\title{
In search of optimal genital herpes management and standard of care (INSIGHTS): doctors' and patients' perceptions of genital herpes
}

\author{
B Romanowski, ${ }^{1}$ Y M Zdanowicz, ${ }^{2}$ S T Owens ${ }^{2}$
}

${ }^{1}$ University of Alberta,

Edmonton, Alberta, Canada;

${ }^{2}$ Ipsos Reid, Toronto, Ontario,

Canada

Correspondence to:

Dr B Romanowski, 1000-8215

112 Street, Edmonton, T6G 2C8,

Alberta, Canada; broman@

docromanowski.com

Presented in part at the 17th International Society for Sexually Transmitted Disease Research, Seattle, Washington, USA, July 2007, poster 395

Accepted 17 September 2007

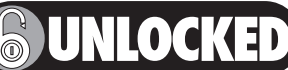

This paper is freely available online under the BMJ Journals unlocked scheme, see http:// sti.bmj.com/info/unlocked.dtl

\begin{abstract}
Objective: To compare and contrast attitudes and behaviours of family doctors and patients with regard to genital herpes and its management.
\end{abstract}

Methods: Family doctors and infected patients were surveyed online to explore disease importance/seriousness, emotional impact, transmission and treatment. The study received ethics approval.

Results: 400 patients and 200 doctors participated. Doctors estimated the emotional impact of genital herpes to be higher than did patients. Patient distress increased with recurrences and more recent diagnosis. Doctors and patients underestimated the risk of transmission during periods of asymptomatic viral shedding, $45 \%$ and $51 \%$, respectively. Doctors reported that $74 \%$ of their patients were taking medication, whereas only $29 \%$ of patients reported use of anti-virals. Doctors reported discussing suppressive therapy with $59 \%$ of patients, whereas only $25 \%$ of patients recalled such a discussion. Only $40 \%$ of patients were aware that daily anti-viral therapy was available to reduce the risk of transmission. The most compelling reason for high interest in suppressive therapy was to reduce the frequency or severity of outbreaks (62\%).

Conclusions: Although doctor and patient attitudes and behaviours coincide in a number of areas, there are many areas of misalignment. This presents opportunities for education and improvement in the management of genital herpes.

Genital herpes, a recurrent life-long infection caused by herpes simplex virus type 1 (HSV-1) and type 2 (HSV-2), is one of the most prevalent sexually transmitted infections in industrialised countries. ${ }^{1-3}$ It causes considerable morbidity, increases the risk of HIV acquisition and transmission, and may lead to congenital and neonatal infections. ${ }^{45}$ Despite increasing awareness of these infections, the prevalence of genital herpes continues to increase, ${ }^{6}$ most marked by an increase in genital HSV-1, and most people with genital herpes are undiagnosed because they are asymptomatic or have unrecognised disease. ${ }^{1}$ Infected people are known to shed virus asymptomatically from the genital area at variable frequencies, ${ }^{7}$ and transmission of genital herpes largely occurs during these periods of asymptomatic shedding. ${ }^{.}$Daily treatment reduces viral shedding and the frequency of recurrences, ${ }^{1}$ and daily treatment with valacyclovir has also been shown to reduce transmission. ${ }^{9}$ Proper condom use can also reduce transmission, and disclosing HSV status to partners before any sexual contact has been proved to lengthen the time to disease transmission. ${ }^{10} 11$
Although many studies into the clinical and psychosexual aspects of genital herpes have been conducted, ${ }^{12-18}$ few have focused on disease perceptions. ${ }^{19-21}$ Published data emphasise the stigma and embarrassment felt by patients: they have difficulty accepting the diagnosis and are most concerned about transmitting genital herpes to current and future sexual partners. Further, patients believe that healthcare professionals are knowledgeable and equipped to diagnose and manage genital herpes. ${ }^{18}$ However, most doctors regard genital herpes as a minor disease that is easy to manage. ${ }^{18}$ Doctors also tend to lack appreciation of the impact of a diagnosis of genital herpes on sexual relationships. ${ }^{18}$

There is a compelling need to document and understand patients' and doctors' perceptions about genital herpes and use the results to improve the management of genital herpes.

This study sought to compare and contrast the attitudes and behaviours of family doctors and infected patients with regard to genital herpes.

\section{METHODS}

Canadian family doctors (doctors) and people with genital herpes (patients) were surveyed using a cross-sectional, online, survey methodology. The survey was administered by Ipsos Reid; data were collected from 15 May to 9 June 2006.

Patients were recruited from Ipsos Reid's consumer panel of over 165000 Canadians. Panellists are amassed from approximately 25 different online sources and all have agreed to participate in research. To achieve a target sample of 400 , a total of 28572 people were sent email invitations, and 13897 (49\%) agreed to participate. Of these, $401(2.9 \%)$ qualified for the study by indicating that they had been diagnosed with genital herpes by a medical doctor or healthcare practitioner.

Doctors were recruited from an email database of 6529 Canadian family doctors. The database comprised email addresses from the Canadian Medical Association Directory as well as doctors previously contacted by Ipsos Reid who had agreed to participate in online research. Recruitment was stratified by geographical region. To achieve a target sample of 200 , a total of 1200 family doctors were invited to participate in the study; 246 were screened, and $200(25 \%)$ in active clinical practice completed the survey. Doctors who indicated having no patients with genital herpes in their practice or those who could not provide an estimate of the number of patients with genital herpes in their practice $(n=49)$ had demographic 
information collected and were only asked several general knowledge questions. The base for the majority of survey questions was therefore 151.

Where possible, the content of the doctor and patient questionnaires mirrored one another. The surveys took $15 \mathrm{~min}$ to complete and covered four main themes related to genital herpes: perceived importance/seriousness, emotional impact, risk of transmission and treatment. Study participants received nominal financial compensation (doctors, \$C65; patients, $\$ C 10)$. The study was approved by the research ethics board of the University of Alberta. All statistical tests were performed using SPSS 12.0.2. software (SPSS Base 12.0 User's Guide, SPSS 2003). Independent sample $t$-tests (assuming unequal variances) were performed when differences between groups, mean values and proportions were tested for. The $\chi^{2}$ test was used to test for differences between groups when variables with multiple categories were examined.

\section{RESULTS}

Doctors had been in practice for 14.7 years (mean) and had 2180 patients (mean) in their practice, with 139 or $6.4 \%$ (mean) diagnosed with genital herpes. Sixty-four per cent of the doctors were male, and $68 \%$ practiced in a city with a population exceeding 100 000. Participating doctors did not differ significantly in terms of gender or language from family doctors listed in the Canadian Medical Directory, but they had been in practice for a shorter period of time (14.7 vs 22.3 years, respectively; $p<0.001$ ).

The mean patient age was 44.4 years and $40 \%$ were male. Age at diagnosis was 31.2 years (mean), and the mean disease duration was 13.2 years; $10 \%$ had had genital herpes for 1 year or less, $13 \%$ for $2-4$ years, $25 \%$ for $5-10$ years, 30\% for $11-20$ years, and $22 \%$ for $20+$ years. Overall, patients had experienced 2.3 outbreaks (mean) in the preceding year, 35\% had had no outbreaks, $36 \%$ one to two, $19 \%$ three to five, and $11 \%$ six or more. Most patients were either married (46\%) or had partners (21\%). Sixty-seven per cent of patients were currently in sexual relationships, $22 \%$ were not sexually active, and $4 \%$ were entering new sexual relationships. Fifty-six per cent lived in a city of over 100000 people, and 19\% in a city of 30000 to just under 100000 .

\section{Diagnosis}

Patients reported that their diagnosis of genital herpes was made by a family doctor in $74 \%$ of cases, $19 \%$ were diagnosed by a specialist, and the remainder were not categorised. Doctors reported that a mean of $40 \%$ of their patients with genital herpes were diagnosed by clinical assessment only, $48 \%$ by genital culture, $6 \%$ using a "blood test", and the remaining $6 \%$ did not specify the method of diagnosis. In contrast, $70 \%$ of patients reported that a genital swab was carried out to confirm their diagnosis, 14\% reported that specific blood tests for herpes were performed, and $24 \%$ stated no test was carried out. A proportion of patients reported more than one method of diagnosis.

Doctors and patients were asked what percentage of people with genital herpes are unaware of their infection. Both groups underestimated the magnitude (doctors, 44\%; patients, $36 \%$; $\mathrm{p}<0.01)$.

\section{Emotional impact of genital herpes}

Doctors and patients acknowledged the negative emotional impact at diagnosis and the ongoing psychological distress related to genital herpes. On average, doctors estimated the emotional impact to be higher than that reported by patients (table 1). Patients with genital herpes for more than 10 years reported less distress than those diagnosed more recently. Distress tended to increase significantly with the number of recurrences in the preceding year.

\section{Transmission of genital herpes}

Patients were asked to indicate sources of infection from a list of options. Virtually everyone cited sexual contact (99\%): however, some considered toilet seats (19\%), blood transfusions $(18 \%)$, shaking hands (8\%), and swimming pools or baths $(6 \%)$ as potential sources of infection. Overall only $65 \%$ identified sexual contact as the sole means of transmission.

Patients estimated that $51 \%$ (mean) of people are infected by a partner who does not have an outbreak at the time of contact, and doctors estimated that $45 \%$ (mean) of cases are transmitted during periods of asymptomatic shedding $(p<0.001)$.

Patients were asked to indicate if a series of statements were true or false. Seventy per cent felt that "genital herpes can be passed to a sexual partner even when you don't have an outbreak". However, 38\% then believed "genital herpes can only be passed to a partner when an infected person has sexual contact during an outbreak" and 25\% believed that "when you are experiencing an outbreak of genital herpes, your sexual partner will be fully protected from contracting the virus if you use a condom". Finally, only $40 \%$ were aware that "there is a daily treatment available to reduce the chances of passing the virus on to your sexual partner".

Doctors and patients were asked to indicate their level of agreement with statements related to transmission (table 1). Over $80 \%$ of patients agreed that "I can handle the outbreaks of genital herpes, it's the risk of transmitting the virus that really troubles me". Doctors were much less likely (62\%) to acknowledge that most patients are more concerned with transmission than outbreaks. Recently diagnosed patients reported more concern with transmission and were more likely to believe that genital herpes can be totally controlled by treating each outbreak than those who had had genital herpes longer. Patients who experienced more outbreaks expressed greater concern about transmission, more disruption to their sex life, and less faith in either condoms or treatments than those with fewer recurrences.

When questioned about strategies for reducing the risk of transmission, doctors reported that they typically suggest consistent condom use (87\%), always informing partners before becoming sexually involved (85\%), abstaining from sexual contact during outbreaks (82\%), taking prescription medication (74\%), and/or using condoms (40\%). Patients, however, typically relied on abstinence during outbreaks (61\%) as opposed to always informing partners before becoming sexually involved (22\%). They also reported consistent condom use $(17 \%)$ or, taking prescription medication during outbreaks $(22 \%)$ or using condoms (15\%) to reduce transmission.

\section{Doctor consultation}

Virtually all doctors (97\%) reported that they typically see their patients with genital herpes at least once a year to discuss the disease. In contrast, only $30 \%$ of patients reported having discussed genital herpes with their doctor within the preceding year. The likelihood of discussions in the preceding year decreased with years since diagnosis and increased with the number of recurrences in the preceding year (fig 1). 
Hypothesis

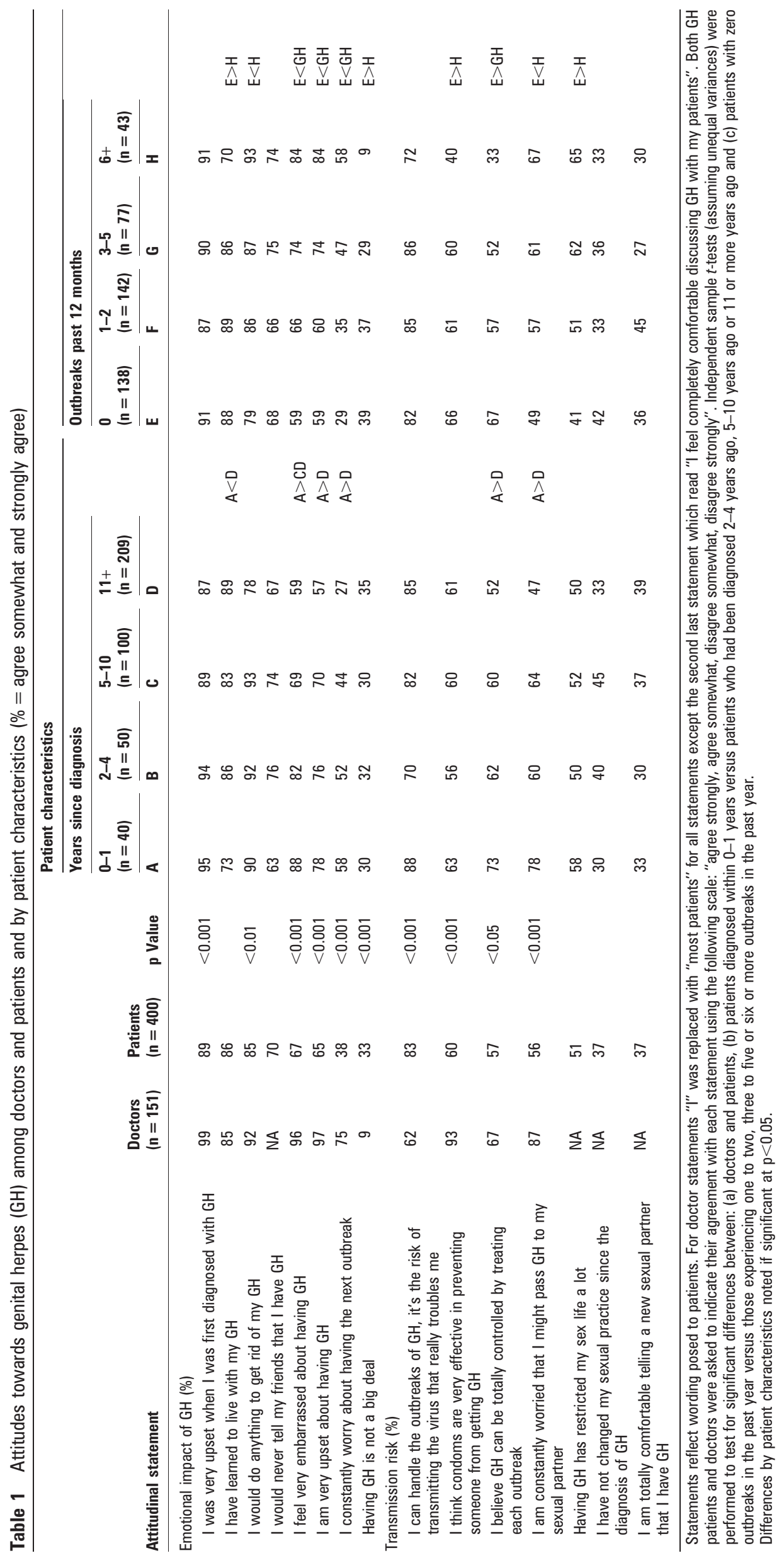

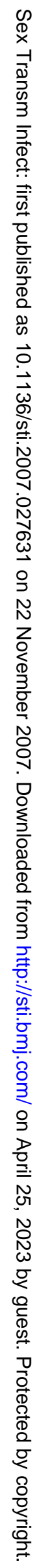

Sex Transm Infect 2008;84:51-56. doi:10.1136/sti.2007.027631

53 
Figure 1 Proportion of patients (\%) visiting a doctor one or more times in the preceding 12 months to discuss genital herpes.

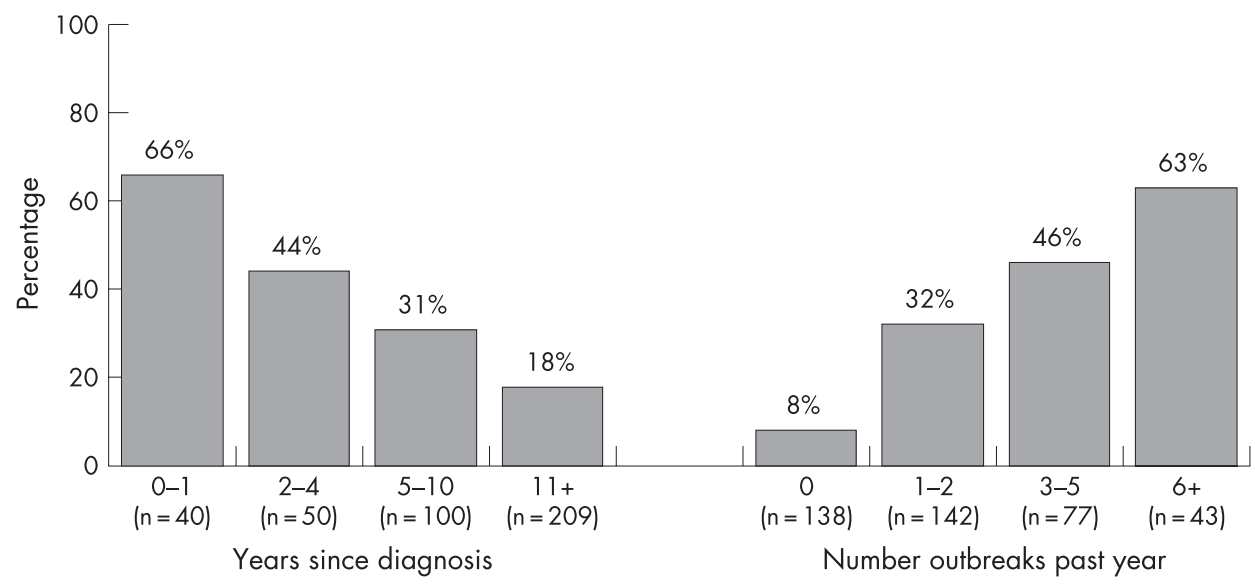

Doctors and patients have different perceptions of who initiated the discussions. According to doctors, 55\% of the discussions were patient-initiated. Among patients who had discussed genital herpes with their doctor, 79\% stated that they initiated the last discussion (fig 2). Patients diagnosed 11+ years ago were more likely to have initiated the last discussion than those diagnosed in the preceding year. Patients experiencing outbreaks in the preceding year were more likely to have initiated the last discussion than those not experiencing outbreaks.

\section{Medical management of genital herpes}

Doctors reported that a mean of $56 \%$ of their patients with genital herpes were currently receiving episodic anti-viral treatment, $18 \%$ were receiving suppressive therapy, and $26 \%$ were not taking prescription medication. In contrast, $20 \%$ of patients reported that they were currently receiving episodic treatment, 9\% suppressive therapy, and $71 \%$ were not taking prescription medication for genital herpes. Use of prescription medication tended to increase with the number of recurrences experienced in the preceding year (table 2).

Among doctors $(n=113)$ who chose not to treat select patients with anti-viral therapy, cost $(42 \%)$ was the primary rationale, followed by patient refusal (26\%) and infrequent outbreaks (13\%) (unaided response). In contrast, patients who were not receiving anti-viral therapy $(n=286)$ thought that their outbreaks were not severe or frequent enough to warrant treatment $(70 \%)$. Cost was only mentioned by $9 \%$ of patients (unaided).

Doctors reported discussing suppressive therapy with 59\% (mean) of patients, whereas only $25 \%$ of patients recalled such a discussion. Among patients, the likelihood of having a discussion about suppressive therapy with a doctor decreased with years since diagnosis and increased with the number of recurrences experienced in the preceding year (table 2).

Doctors were asked to rank a list of factors in terms of their importance in determining whether suppressive therapy is appropriate (table 3). Reducing the frequency of outbreaks (81\%) was most important followed by reducing the risk of transmission (71\%) and reducing the severity of each outbreak (58\%).

Doctors reported the following reasons for not prescribing suppressive therapy: cost (47\%), infrequent outbreaks (38\%) and poor compliance (30\%) (unaided). Patients who had discussed suppressive therapy but who were not receiving it ( $n=63$ ) cited infrequent or mild outbreaks (49\%), the cost of treatment or lack of drug coverage (25\%), doctor didn't feel that therapy was warranted (24\%), and concern about side effects $(24 \%)$ as reasons for not starting suppressive therapy (unaided).

Doctors and patients were asked about patients' willingness to start suppressive therapy (table 4). Seventy-two per cent of doctors felt that patients are very or somewhat willing to start

Figure 2 Who initiated last discussion of genital herpes as reported by patients.

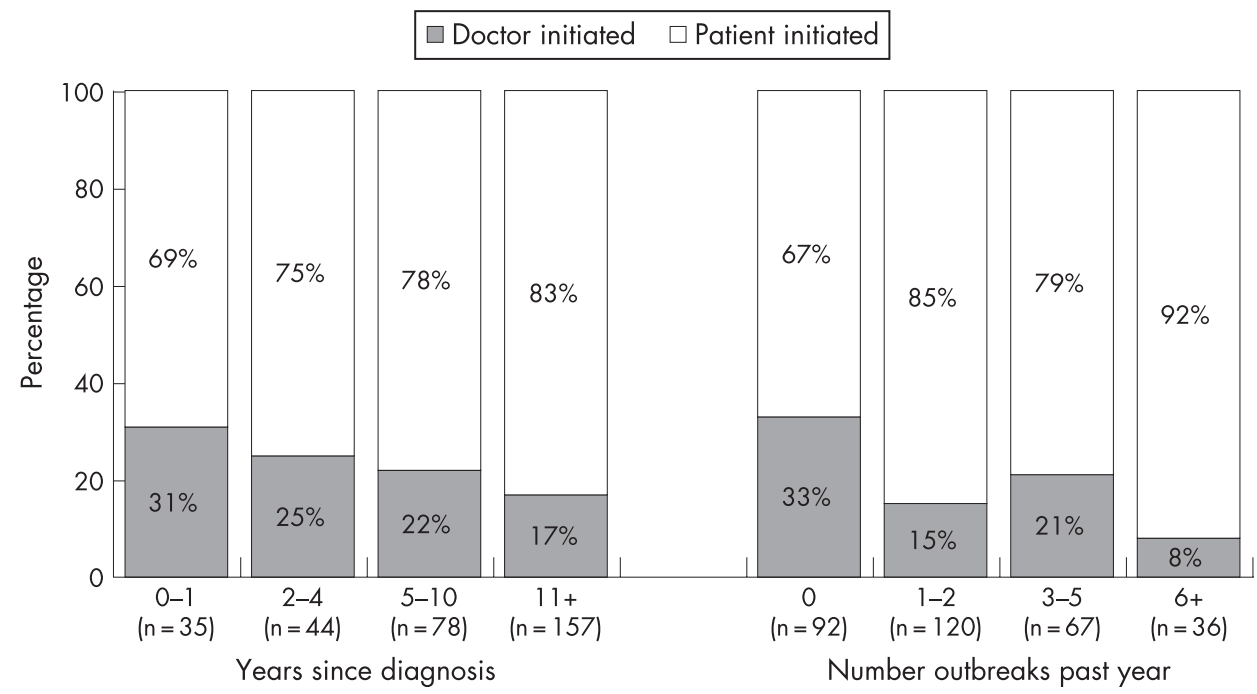


Table 2 Medical management of genital herpes by patient characteristics (\%)

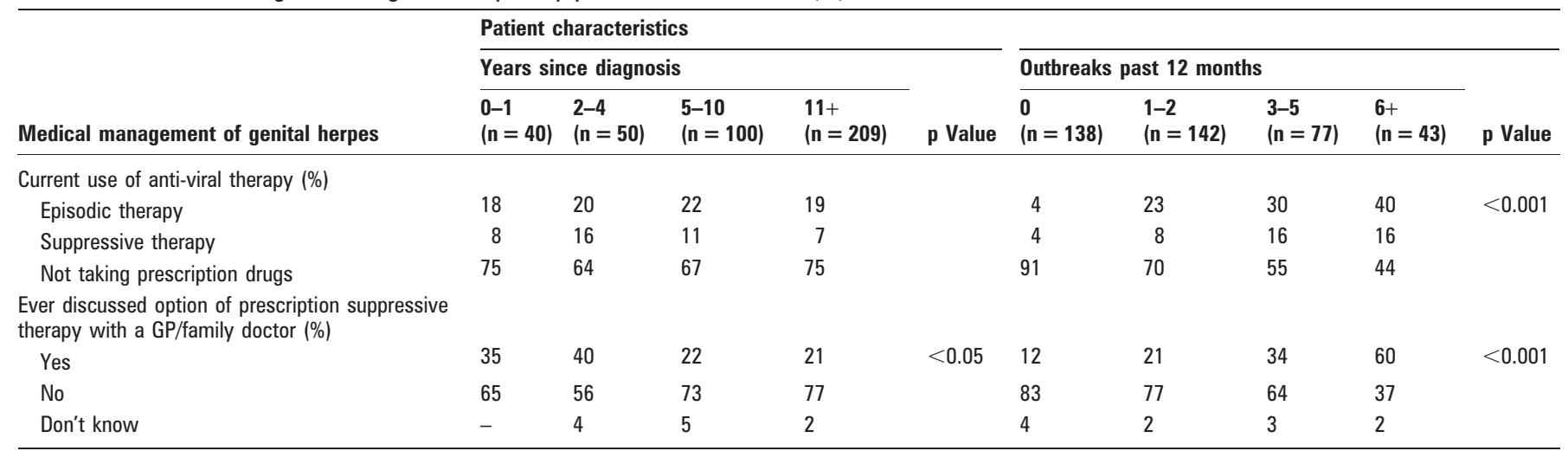

Differences across years since diagnosis and outbreaks tested using $\chi^{2}$ test.

Table 3 Factors that doctors might consider when determining if suppressive therapy is appropriate (\% ranked among top three factors)

\begin{tabular}{ll}
\hline To reduce the frequency of outbreaks & 81 \\
To reduce the likelihood of transmitting the virus & 71 \\
To reduce the severity of each outbreak & 58 \\
To reduce the duration of each outbreak & 34 \\
To reduce the psychological distress of recurrent outbreaks to the patient & 30 \\
To reduce the psychological distress of the risk of transmitting genital & 25 \\
herpes to a partner &
\end{tabular}

herpes to a partner

Values are the number of doctors out of 151 who considered the factor when determining if suppressive therapy is appropriate.

suppressive therapy versus $52 \%$ of patients not already receiving suppressive therapy. Patient willingness to receive suppressive therapy increased with the number of outbreaks experienced in the preceding year.

The most compelling reason for high interest in suppressive therapy $(n=99)$ was to reduce the frequency or severity of outbreaks $(62 \%)$ followed by only $11 \%$ of patients who cited having treatment to reduce the risk of transmission (unaided). Patients not willing to receive suppressive therapy $(\mathrm{n}=142)$ felt that their outbreaks were not severe or frequent enough $(63 \%)$, were reluctant to take any drugs (18\%), and felt they could handle the outbreaks without drugs $(16 \%)$. Concerns about side effects $(7 \%)$ and cost of drugs (6\%) were far less salient (unaided).

\section{DISCUSSION}

This survey compares and contrasts attitudes and behaviours of family doctors and patients with regard to genital herpes. It identifies notable gaps that have relevance for improving educational efforts and the management of genital herpes. The general knowledge of doctors and patients about the diagnosis, asymptomatic shedding and risk of transmission of genital herpes was disappointing. Clearly, there continues to be a need for education about the basic facts of this infection.

The 2006 Canadian Sexually Transmitted Infections Guidelines $^{22}$ stress the importance of "sensitive, empathetic, knowledgeable counselling" as a component in improving the physical and psychological morbidity associated with genital herpes. Our study confirms that genital herpes carries a physical and emotional burden, and patients are more concerned with the risk of transmitting the virus than they are with recurrences. However, not all individuals experience the same degree of morbidity. Our results and those of others confirm that emotional distress is more pronounced among those with more recurrences, and, over time, people cope better with genital herpes. ${ }^{161820}$ Doctors are aware of the negative psychological impact of genital herpes and reported that their patients experienced greater emotional impact than did the patients themselves. It is possible that, when evaluating emotional harm, doctors tended to think about newly diagnosed patients or those who experienced a greater number of outbreaks.

Doctors and patients differed in their accounts of how genital herpes was managed in the primary care setting. Doctors felt that they were more proactive and provided more information, especially on treatment and the risk of transmission, than was reported by patients. Perhaps information is being delivered, but patients do not comprehend the messages.

Both doctors and patients underestimated the risk of transmission during periods of asymptomatic viral shedding, $45 \%$ and $51 \%$, respectively, versus $70 \%$ reported in the literature. ${ }^{83}$ This lack of information is reflected in patients' behaviour of predominantly relying on abstinence during outbreaks to reduce the risk of transmission. Education that focuses on a better understanding of asymptomatic viral shedding and transmission reduction strategies is important.

There was also substantial disparity in the reported use of anti-viral therapy between doctors and patients. It is possible

Table 4 Patient willingness to receive suppressive therapy (\%)

\begin{tabular}{|c|c|c|c|c|c|c|c|c|c|c|c|c|}
\hline \multirow[b]{3}{*}{ Patient willingness } & \multirow[b]{3}{*}{$\begin{array}{l}\text { Doctors } \\
\text { (n= 151) }\end{array}$} & \multirow[b]{3}{*}{$\begin{array}{l}\text { Patients* } \\
\text { (n= 364) }\end{array}$} & \multirow[b]{3}{*}{ p Value } & \multicolumn{9}{|c|}{ Patient characteristics } \\
\hline & & & & \multicolumn{4}{|c|}{ Years since diagnosis } & \multicolumn{4}{|c|}{ Outbreaks past 12 months } & \multirow[b]{2}{*}{ p Value } \\
\hline & & & & $\begin{array}{l}0-1 \\
(n=37)\end{array}$ & $\begin{array}{l}2-4 \\
(n=42)\end{array}$ & $\begin{array}{l}5-10 \\
(n=89)\end{array}$ & $\begin{array}{l}11+ \\
(n=195)\end{array}$ & $\begin{array}{l}0 \\
(n=132)\end{array}$ & $\begin{array}{l}1-2 \\
(n=131)\end{array}$ & $\begin{array}{l}3-5 \\
(n=65)\end{array}$ & $\begin{array}{l}6+ \\
(n=36)\end{array}$ & \\
\hline Extremely/very willing & 18 & 28 & $<0.001$ & 46 & 21 & 33 & 23 & 14 & 31 & 31 & 58 & $<0.001$ \\
\hline Somewhat willing & 54 & 24 & & 11 & 24 & 25 & 27 & 17 & 29 & 32 & 22 & \\
\hline Not at all/not very willing & 28 & 39 & & 30 & 45 & 31 & 43 & 55 & 33 & 29 & 19 & \\
\hline Don't know & 1 & 9 & & 14 & 10 & 11 & 8 & 14 & 8 & 8 & 0 & \\
\hline
\end{tabular}

\footnotetext{
${ }^{*}$ Among patients who are not currently receiving suppressive therapy. All tests for group differences conducted using $\chi^{2}$ test.
} 
that episodic therapy was under-reported given the focus on drugs "currently" being taken to treat genital herpes. Patients might not have considered themselves "current users" if they were only taking anti-viral drugs with each recurrence.

Treatment guidelines ${ }^{22}$ acknowledge the benefits of suppressive therapy for the prevention of recurrent outbreaks, reduction of asymptomatic shedding, and valacyclovir specifically to reduce the risk of transmission. ${ }^{9}$ Other research suggests that patients report a strong preference for suppressive therapy, independent of the reduction in transmission. ${ }^{25}$ However, in our study only $9 \%$ of patients were receiving suppressive therapy. Doctors reported discussing daily treatment with many of their patients, but few patients recalled such a discussion. Moreover, most patients said that they were willing to start suppressive therapy. For doctors and patients, use of suppressive therapy centred on outbreak reduction rather than reducing the risk of transmission. Many patients were not considered for suppressive therapy because their morbidity was not "severe enough". However, only $16 \%$ of patients experiencing six or more outbreaks in the preceding year were receiving daily anti-viral therapy. Another reason doctors cited for not prescribing antiviral drugs was cost, but this was reported as an impediment by very few patients. These results are similar to those reported by others ${ }^{18} 20$ and demonstrate a large discrepancy between knowledge, guideline recommendations, and management practices.

Potential limitations of our study include the subject sampling source, which may have resulted in the inclusion of subjects who were more willing to discuss genital herpes and were more engaged in treatment. The online consumer panel was chosen because it provided a large sample source with high response rates, providing an economical means of reaching members of this population in a "safe" and anonymous atmosphere. Internet-based research does not allow matching of doctors and patients, but we believe that our sample is representative of the two populations studied. A similar recruitment strategy has been used by others. ${ }^{17} 1820$

\section{CONCLUSION}

Education for doctors and patients needs to clarify the efficacy of available strategies to reduce asymptomatic shedding and the risk of transmission including anti-viral therapy and condoms. This can be achieved only if patients are empowered with information and are able to have an informed and constructive discussion with their doctor. Doctors recognise the emotional impact of genital herpes and believe that they are providing patients with choices on disease management. However, patients are not recognising these choices. Doctors must ensure that they are accurate and complete in their knowledge of genital herpes and adapt their communication style so that choices in disease management are truly shared with patients. The challenge will be to educate both groups, with the goal of achieving optimal genital herpes management and standard of care as the norm not the exception. This standard of care should include a laboratory-confirmed diagnosis, ongoing education and discussion of genital herpes including basic disease information, asymptomatic viral shedding and risk of transmission. Counselling and appropriate recommendations about treatment based on morbidity, the psychological impact of genital herpes and risk of transmission to a partner are also important components within this standard of care. The information must be conveyed in an appropriate format to ensure that it is received and understood by patients with genital herpes.

Acknowledgements: We thank Bernie Muise of GlaxoSmithKline for helpful discussions and input throughout the study.

Funding: Financial support: GlaxoSmithKline Inc, Canada

Competing interests: BR is a consultant for GlaxoSmithKline and has received research funds and honoraria for speaking engagements from them. YMZ and STO are employed by an independent market research company and have received consulting fees from GlaxoSmithKline to conduct the fieldwork for this study as well as to consult on the methodology, analysis and preparation of the journal article.

\section{REFERENCES}

1. Kimberlin DW, Rouse DJ. Genital herpes. N Engl J Med 2004;350:1970-7.

2. Nahmias AJ, Lee FK, Beckman-Nahmias S. Sero-epidemiological and sociological patterns of herpes virus infection in the world. Scand J Infect Dis Supp/ 1990;69:1936.

3. Smith JS, Robinson NJ. Age-specific prevalence of infection with herpes simplex virus types 2 and 1: a global review. J Infect Dis 2002;186:S3-28.

4. Wald A, Link K. Risk of human immunodeficiency virus infection in herpes simplex virus type-2 seropositive persons: a meta analysis. J Infect Dis 2002;185:45-52.

5. Brown ZA, Selke S, Zeh J, et al. The acquisition of herpes simplex virus during pregnancy. N Engl J Med 1997;337:509-15.

6. Xu F, Sternberg MR, Kottiri BJ, et al. Trends in herpes simplex virus type 1 and type 2 seroprevalence in the United States. JAMA 2006;296:964-973.

7. Wald A, Zeh J, Selke $S$, et al. Reactivation of genital herpes simplex virus type 2 infection in asymptomatic seropositive persons. N Engl J Med 2000;342:844-50.

8. Mertz GJ, Benedetti J, Ashley R, et al. Risk factors for the sexual transmission of genital herpes. Ann Intern Med 1992;116:197-202

9. Corey L, Wald A, Patel R, et al. Once daily valacyclovir to reduce the risk of transmission of genital herpes. N Engl J Med 2004;350:11-20.

10. Wald A, Langenberg AG, Link K, et al. Effect of condoms on reducing the transmission of herpes simplex virus type 2 from men to women. JAMA 2001;285:3100-6.

11. Wald A, Krantz E, Selke S, et al. Knowledge of partner's genital herpes protects against herpes simplex virus type 2 acquisition. $J$ Infect Dis 2006;194:42-52.

12. Mindel A. Psychological and psychosexual implications of herpes simplex virus infections. Scand J Infect Dis Supp/ 1996;100:27-32.

13. Brookes JL, Haywood S, Green J. Adjustment to the psychological and social sequelae of recurrent genital herpes simplex infection. Genitourin Med 1993;69:3847.

14. Goldmeier D, Johnson A, Byrne M, et al. Psychosocial implications of recurrent genital herpes virus infection. Genitourin Med 1988;64:327-30.

15. Levenson JL, Hamer RM, Myers T, et al. Psychological factors predict symptoms of severe recurrent genital herpes infection. J Psychosom Res 1987;31:153-9.

16. Carney 0, Ross E, Bunker C, et al. A prospective study of the psychological impact on patients with a first episode of genital herpes. Genitourin Med 1994;70:40-5.

17. Catotti DN, Clarke P, Catoe K. Herpes revisited. Still a cause for concern. Sex Transm Dis 1993;20:77-80.

18. Alexander L, Naisbett B. Patient and physician partnerships in managing genital herpes. J Infect Dis 2002;186(Suppl 1):S57-65.

19. Patel R, Boselli F, Cairo I, et al. Patients' perspective on the burden of recurrent genital herpes. Int J STD AIDS 2001;12:640-5

20. Gilbert L, Scanlon K, Peterson R, et al. Patient and partner perceptions about preventing genital herpes transmission. Herpes 2005;12:60-5.

21. Gilbert LK, Schulz SL, Ebel C. Education and counselling for genital herpes: perspectives from patients. Herpes 2002;9:78-82.

22. Public Health Agency of Canada. Canadian guidelines on sexually transmitted infections. 2006 edition. Ottawa: Public Health Agency of Canada, 2006.

23. Narouz N, Allan PS, Wade AH. Genital herpes: general practioners' knowledge and opinions. Sex Transm Infect 2002;78:198-200.

24. CDC. Sexually transmitted diseases treatment guidelines. MMWR 2006;55(RR11):194.

25. Romanowski B, Marina RB, Roberts JN. the Valtrex HS230017 Study Group. Patient preference of valacyclovir once daily suppressive therapy versus twice daily episodic therapy for recurrent genital herpes: a randomized study. Sex Trans Dis 2003;30:226-31. 